\title{
Dimorfismo sexual na "cobra-cipó" Oxybelis aeneus (Serpentes, Colubridae) no estado do Ceará, Brasil
}

\author{
Paulo Cesar Mattos Dourado de Mesquita* \\ Diva Maria Borges-Nojosa \\ Castiele Holanda Bezerra \\ Universidade Federal do Ceará \\ Campus do Pici, Bloco 905, CEP 60455-760, Fortaleza-CE, Brasil \\ *Autor para correspondência \\ paulocmdm@gmail.com
}

Submetido em 26/04/2010

Aceito para publicação em 17/09/2010

\section{Resumo}

Diferentes pressões determinam o sucesso reprodutivo de machos e fêmeas e por esta razão espera-se encontrar diferenças morfológicas entre os sexos de qualquer espécie. Oxybelis aeneus é uma serpente arborícola, longa e delgada, amplamente distribuída dos Estados Unidos ao sudoeste do Brasil. A análise de 102 indivíduos forneceu informações sobre dimorfismo sexual nesta espécie. Fêmeas são maiores que machos em relação ao tamanho corpóreo (CRC e massa), mas não foi observado dimorfismo em relação ao comprimento relativo caudal e tamanho da cabeça de machos e fêmeas.

Unitermos: arborícola, dimorfismo sexual, morfologia, serpentes

\section{Abstract}

Sexual dimorphism in the brown vine-snake Oxybelis aeneus from the State of Ceara, Brazil. Several different pressures determine the reproductive success among males and females, and that is the reason why morphological differences between the sexes are expected to be found in any given species. Oxybelis aeneus is a tiny and slender arboreal snake that is widely distributed from the United States to southeastern Brazil. The analysis of 102 specimens provided information on sexual dimorphism for this species. Females are longer and heavier than males, while there is no difference in head size and tail length between males and females.

Key words: arboreal, morphology, sexual dimorphism, snakes

\section{Introdução}

Devido às diferentes pressões que determinam o sucesso reprodutivo de machos e fêmeas, é previsto desde Darwin (1871) que existam diferenças morfológicas entre os sexos de qualquer espécie. Ocasionalmente, como no caso de animais de morfologia simples, como as serpentes, estas diferenças podem não ser explícitas ao observador humano (Bonnet et al., 1998).

O dimorfismo sexual pode se apresentar de diferentes formas entre as serpentes (Rivas e Burghardt, 2001) e para diversas espécies foi comprovado dimorfismo sexual relacionado às dimensões e 
proporções corpóreas (Shine, 1993; 1994), quanto à forma e tamanho da cabeça (Camilleri e Shine, 1990; Shine, 1995; Vincent et al., 2004), ao tamanho de órgãos e glândulas (Kissner et al., 1998) e mais raramente, à coloração (Shine, 1993; Marques e Sazima, 2003).

Algumas serpentes brasileiras, como Philodryas patagoniensis (López e Giraudo, 2008), Leptodeira annulata, Sibynomorphus mikanii, S. neuwiedi (Pizzato et al., 2008), Dipsas neivai e D. catesbyi (Alves et al., 2005) foram analisadas quanto ao dimorfismo sexual e apresentaram fêmeas maiores que machos. Existem, porém, espécies como Imantodes cenchoa, Epicrates cenchria crassus, Chironius bicarinatus, C. flavolineatus que os machos são maiores que as fêmeas, sugerindo a existência de ritual de combate entre machos (Feio et al., 1999; Almeida-Santos e Marques, 2002; Pizzatto et al., 2006; Pizzatto, 2006; Pizzatto et al., 2008).

Oxybelis aeneus é uma serpente arborícola, longa e delgada amplamente distribuída desde o Estado do Arizona nos Estados Unidos até o sudoeste do Brasil (Stebbins, 1954). O propósito deste trabalho é apresentar informações sobre o dimorfismo sexual de Oxybelis aeneus em relação ao comprimento rostro cloacal (CRC), comprimento relativo caudal, comprimento da cabeça e massa.

\section{Material e Métodos}

A amostra foi composta de 63 indivíduos de $O$. aeneus capturados em campo no município de Pentecoste, entre os meses de Janeiro de 2008 a Dezembro de 2009, e outros 39 indivíduos do Estado do Ceará depositados na Coleção de Herpetologia da Universidade Federal do Ceará (CHUFC), totalizando 102 espécimes analisados.

O dimorfismo sexual foi analisado tendo como referência as medições de comprimento rostro-cloacal (CRC, em mm), comprimento relativo da cauda (CC, em $\mathrm{mm}$ ), comprimento da cabeça (HL, em mm) e massa (em mg). Para comparar as medidas de CRC foi realizada uma análise de variância (ANOVA) e o cálculo do Índice de Dimorfismo Sexual em Tamanho (SSD "Sexual Size Dimorphism"):

$$
S S D=\frac{\bar{X} C R C(\text { fêmea })}{\bar{X} C R C(\text { macho })}
$$

onde: $\mathrm{CRC}_{\text {(fêmea) }}=\mathrm{CRC}$ das fêmeas

$\mathrm{CRC}_{\text {(macho) }}=\mathrm{CRC}$ dos machos

Este índice é avaliado a depender do resultado: se $\mathrm{SSD}>0$ = fêmeas maiores que machos e se SSD < $0=$ machos maiores que fêmeas (Shine, 1994). Para comparar as medidas de CC e HL foram realizadas análises de covariância (ANCOVA) utilizando o CRC como covariável. De forma semelhante, para comparar diferenças na massa, foi realizada uma análise de covariância (ANCOVA) utilizando o comprimento total $(\mathrm{CRC}+\mathrm{CC})$ como covariável da raiz cúbica da massa.

Indivíduos que apresentaram amputação parcial ou total da cauda, indivíduos que não foram sexados e neonatais não foram utilizados nas análises que utilizaram os valores de $\mathrm{CC}$ e comprimento total.

Todos os testes foram realizados com auxilio do programa STATISTICA for Windows 7.0 (STATSOFT, 1998) com um grau de confiança $95 \%$.

\section{Resultados}

O CRC das fêmeas variou entre 425 e $1030 \mathrm{~mm}$ $(\bar{X}=846,1 \pm 131,1 ; \mathrm{n}=46)$ enquanto o dos machos variou entre 470 e $1026 \mathrm{~mm}(\bar{X}=793,8 \pm 143,1 ; \mathrm{n}=37)$. As fêmeas apresentaram-se significativamente maiores que os machos em relação ao $\mathrm{CRC}\left(\mathrm{F}_{1,83}=4,279 ; \mathrm{p}=\right.$ 0,042). O índice SSD para esta espécie foi de 0,066, indicando fêmeas um pouco maiores que os machos.

O CC de machos e fêmeas não diferiu significativamente $\left(\mathrm{F}_{1,66}=0,273 ; \mathrm{p}=0,603\right)$ (Figura 1), ou seja, não há dimorfismo sexual no tamanho da cauda em O. aeneus. Também não houve diferença entre o HL de machos e fêmeas $\left(\mathrm{F}_{1,84}=0,594 ; \mathrm{p}=0,443\right)$ (Figura 2).

As fêmeas apresentaram uma massa maior que a dos machos (Figura 3) $\left(\mathrm{F}_{1,78}=114,5 ; \mathrm{p}<0,0001\right)$. 


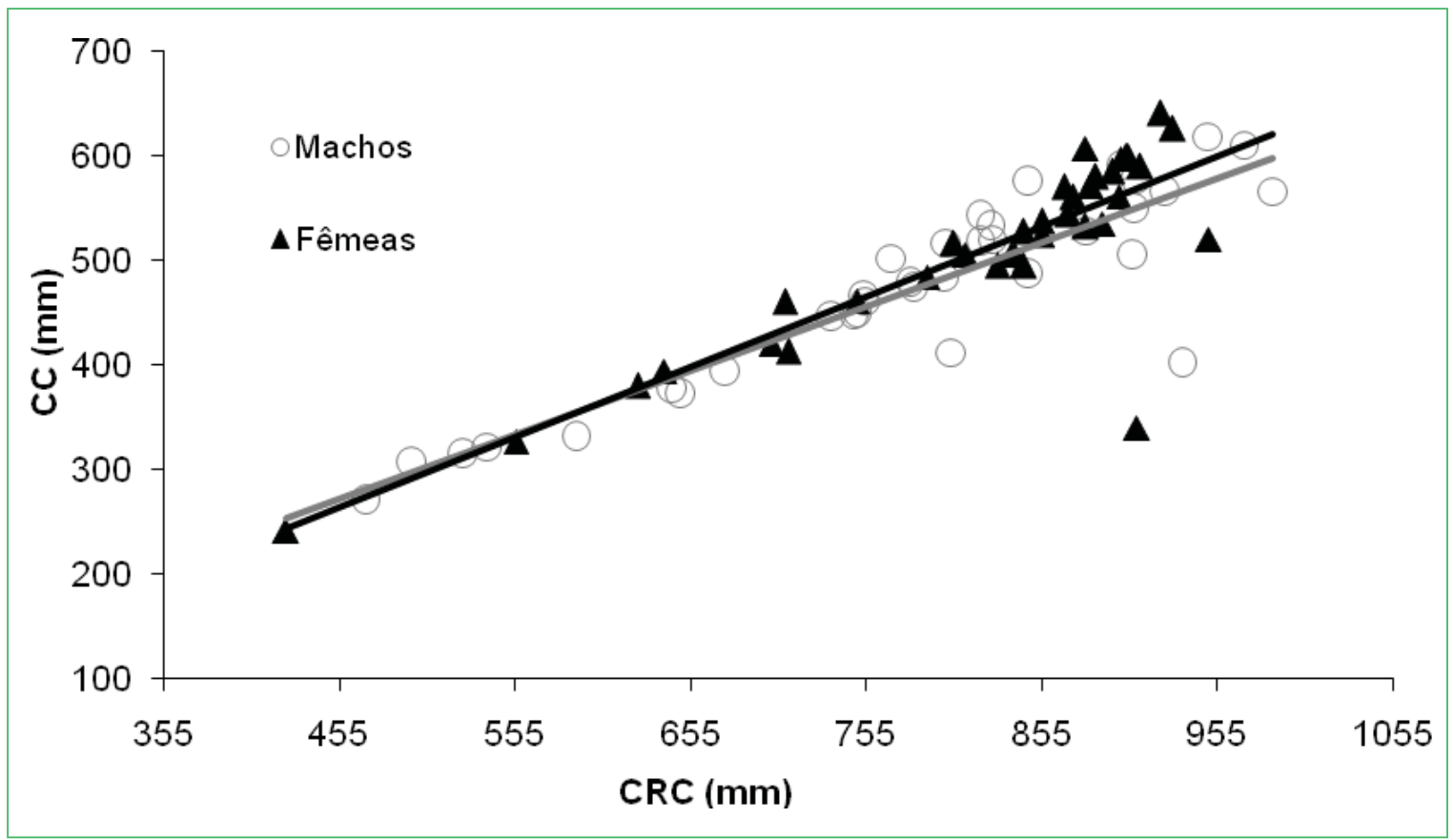

FIGURA 1: Relação CC x CRC de machos e fêmeas de Oxybelis aeneus. Círculos = machos; Triângulos = fêmeas.

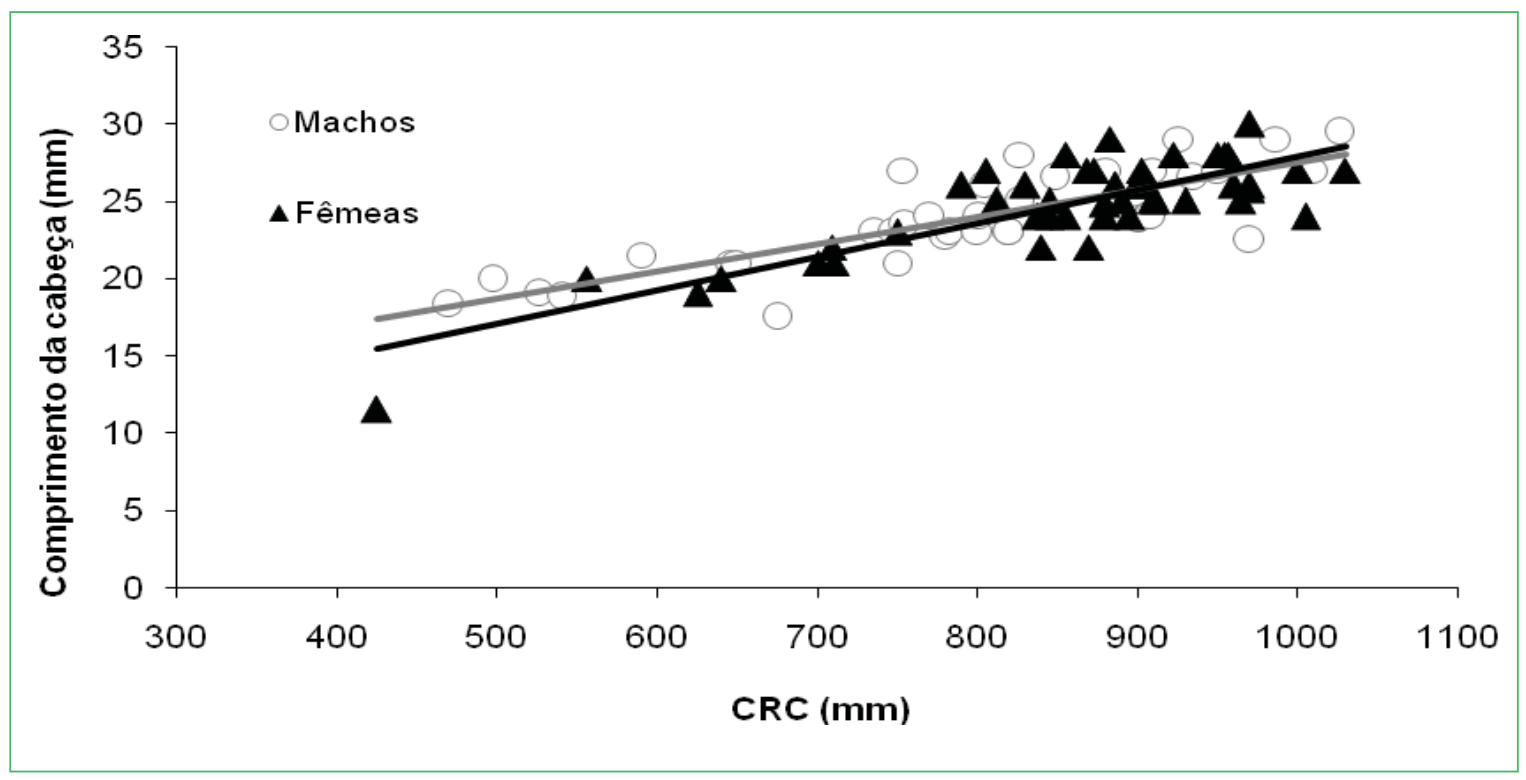

FIGURA 2: Relação Comprimento da Cabeça x CRC de machos e fêmeas de Oxybelis aeneus. Círculos = machos; Triângulos $=$ fêmeas. 


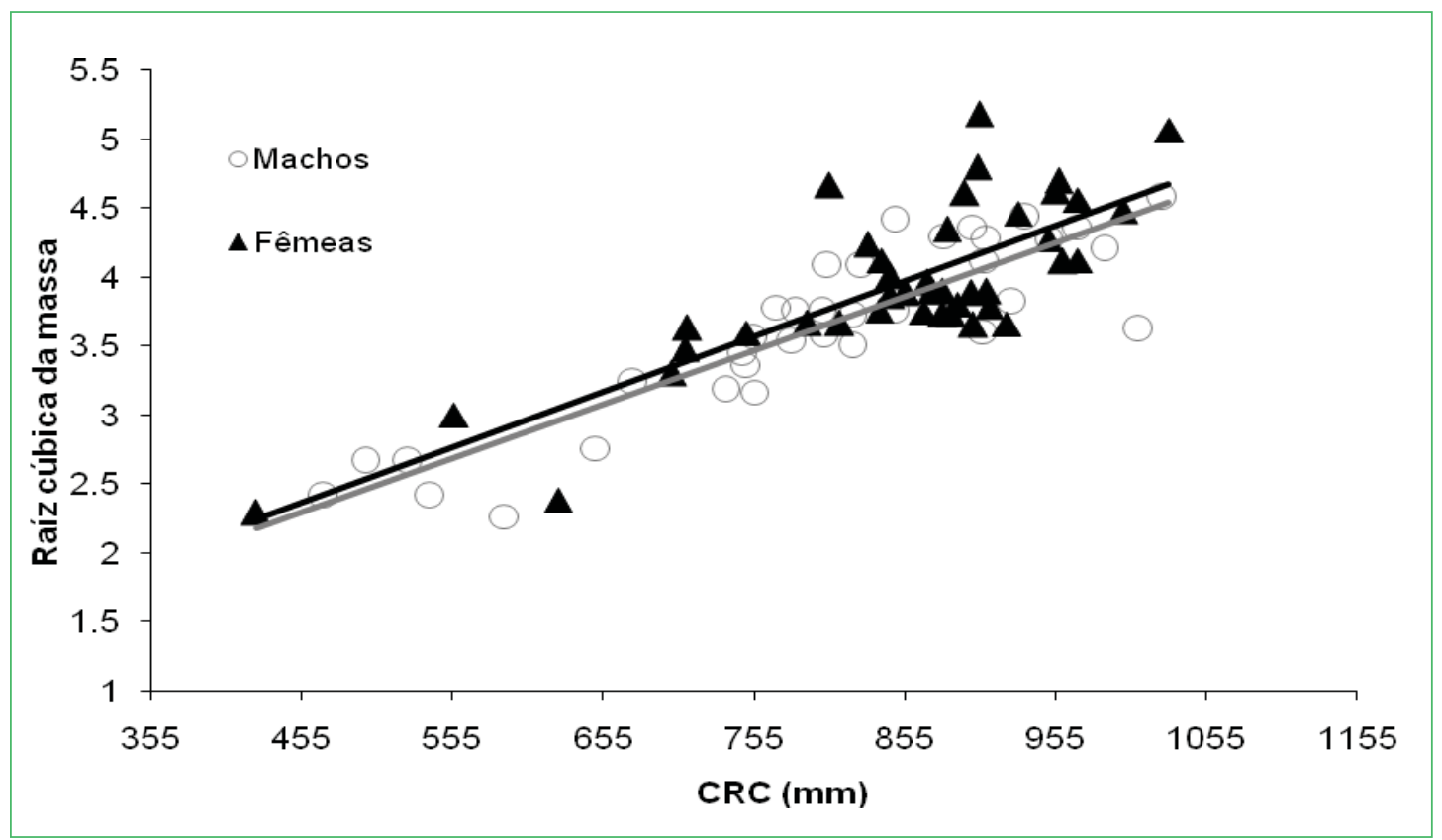

FIGURA 3: Relação Raíz cúbica da massa x CRC de machos e fêmeas de Oxybelis aeneus. Círculos = machos; Triângulos $=$ fêmeas.

\section{Discussão}

Diferenças sexuais no tamanho do corpo de serpentes são consideradas adaptações que podem representar um maior sucesso reprodutivo (Pizzatto et al., 2007). Por isso, fêmeas apresentando um tamanho maior do que os machos é um padrão comumente encontrado entre colubrídeos (Shine, 1993; 1994). Como o sucesso reprodutivo das fêmeas está diretamente relacionado com a quantidade e com o tamanho dos ovos que são capazes de gerar a cada ciclo, o maior tamanho corporal das fêmeas deve estar relacionado com a fecundidade. Assim, espera-se que a seleção natural favoreça fêmeas maiores (Pizzato et al., 2007). Em adição a isto, o dimorfismo sexual em relação ao CRC é uma evidência de que machos e fêmeas maturam em diferentes tamanhos, uma vez que a taxa de crescimento é reduzida após a maturidade sexual. Portanto os machos devem atingir a maturidade sexual em um tamanho menor que as fêmeas.

Para a maioria dos colubrídeos os machos apresentam caudas relativamente mais longas que as fêmeas devido à presença do hemipênis, dos músculos relacionados ao órgão copulador que ficam localizados na base da cauda (Klauber, 1972; King, 1989) e ao aumento da eficiência durante a cópula, supostamente machos com caudas maiores levam vantagem em rituais de disputa por fêmeas (Kaufman e Gibbons, 1975; Shine et al., 1999). Entretanto a ausência de dimorfismo em relação ao $\mathrm{CC}$ em $O$. aeneus deve se dar devido a pressões não meramente sexuais. A diminuição do dimorfismo em relação ao comprimento caudal em $O$. aeneus pode ter se dado devido à pressão evolutiva em relação ao uso do ambiente, provenientes das vantagens conferidas por uma cauda mais longa no equilíbrio do corpo e na locomoção em ambientes arbóreos (Pizzatto et al. 2007).

Em geral as fêmeas de colubrídeos apresentam uma massa maior que a dos machos (Shine, 1994). Isto está diretamente relacionado com o tamanho corpóreo das fêmeas, que, em geral, é superior ao dos machos e a pressão em relação à produção de ovos deve favorecer fêmeas maiores. Os resultados encontrados para $O$. aeneus neste estudo são consistentes com esta hipótese. Cunha e Nascimento (1993) comentam brevemente que o dimorfismo sexual nesta espécie seria pouco significativo, idéia refutada pelos dados obtidos aqui, pois apesar de pouco pronunciado ao observador humano o dimorfismo existe.

Diferenças entre o tamanho da cabeça entre machos e fêmeas de serpentes geralmente estão relacionadas 
à segregação alimentar, com cada um dos sexos se especializando em um determinado tipo ou tamanho de presa diferente (Luiselli et al., 2002). A não existência de dimorfismo sexual neste sentido sugere que ambos os sexos se alimentam de presas similares, porém, estudos sobre a dieta desta espécie devem ser realizados para confirmar esta sugestão.

\section{Referências}

Almeida-Santos, S. M.; Marques, O. A. V. 2002. Male-male ritual combat in the colubrid snake Chironius bicarinatus in the Atlantic forest, southeastern Brazil. Amphibia-Reptilia, 23: 528-533.

Alves, F. Q.; Argolo, A. J. S.; Jim, J. 2005. Biologia reprodutiva de Dipsas neivai Amaral e D. catesbyi (Sentzen) (Serpentes, Colubridae) no sudeste da Bahia, Brasil. Revista Brasileira de Zoologia, 22 (3): 573-579.

Bonnet, X.; Shine, R.; Naulleau, G.; Vallas-Vacher, M. 1998. Sexual dimorphism in snakes: different reproductive roles favour different body plans. Proceedings of the Royal Society of London, 265: 179-183.

Camilleri, C.; Shine, R. 1990. Sexual dimorphism and dietary divergence: differences in trophic morphology between male and female snakes. Copeia, 1990 (3): 658-665.

Cunha, O. R.; Nascimento, F. P. 1993. Ofídios da Amazônia. As cobras da região Leste do Pará. Boletim do Museu Paraense Emílio Goeldi, Série Zoologia, 9: 1-191.

Darwin, C. 1871. The descent of man and selection in relation to sex. $1^{\text {st }}$ ed. Murray, London, UK. Disponível em $<$ http://darwinonline.org.uk/EditorialIntroductions/Freeman_TheDescentofMan. html>. Acesso em 22 de abril de 2009.

Feio, R.; Santos, P. S; Fernandes, R.; Freitas, T. S. 1999. Chironius flavolineatus. Courtship. Herpetological Review, 30: 99-100.

Kaufman, G. A.; Gibbons, J. W. 1975. Weight-length relationships in thirteen species of snakes in the southeastern United States. Herpetologica, 31: 31-37.

King, R. B. 1989. Sexual dimorphism in tail length: sexual selection, natural selection, or morphological constraint? Biological Journal of Linnean Society, 38: 133-154.

Kissner, K.J.; Forbes, M.R.; Secoy, D. M. 1998. Sexual dimorphism in size of cloacal glands of garter snake, Thamnophis radix haydeni. Journal of Herpetology, 32: 268-270.
Klauber, L. M. 1972. Rattlesnakes their habits, life-histories, and influence of mankind. University of Califonia Press, Berkeley, USA, 740pp.

López, M. S; Giraudo, A. R. 2008. Ecology of the snake Philodryas patagoniensis from Northeast Argentina. Journal of Herpetology, 42 (3): 474-480.

Luiselli, L.; Akani, G. C.; Angelici, F. M. 2002. Comparative feeding strategies and dietary plasticity of the sympatric cobras Naja melanoleuca and Naja nigricollis in three diverging Afrotropical habitats. Canadian Journal of Zoology, 80: 55-63.

Marques, O. A. V.; Sazima, I. 2003. Bothrops jararacussu (Jararacussu). Sexual dichromatism. Herpetological Review, 34: 62.

Pizzatto, L. 2006. Ecomorfologia e estratégias reprodutivas dos Boidae (Serpentes), com ênfase nas espécies neotropicais. Tese de Doutorado, Universidade Estadual de Campinas, Brasil, 151pp.

Pizzatto, L.; Almeida-Santos, S. M.; Shine, R. 2007. Life-History adaptations to arboreality in snakes. Ecology, 88 (2): 359-366.

Pizzatto, L.; Cantor, M.; Oliveira, J. L.; Marques, O. A. V.; Capovilla, V.; Martins, M. 2008. Reproductive ecology of dipsadine snakes, with emphasis on South American species. Herpetologica, 64 (2): 168-179.

Pizzatto, L.; Haddad, R. M.; Almeida-Santos, S. M. 2006. Malemale ritualized combat in the brazilian rainbow boa Epicrates cenchria crassus. Herpetological Bulletin, 95: 16-20.

Rivas, J. A.; Burghardt, G. M. 2001. Understanding sexual size dimorphism in snakes: wearing the snake's shoes. Animal Behaviour, 62: 1-6.

Shine, R. 1993. Sexual dimorphism in snakes. In: Seigel, R.A. \& Collins, J. T. (Eds). Snakes, Ecology and Behavior. McGraw-Hill, New York, USA, p.49-86.

Shine, R. 1994. Sexual size dimorphism in snakes revisited. Copeia, 1994: 326-346.

Shine, R. 1995. Australian snakes: a natural history. Cornell University Press, Itchaca, Australia, 224pp.

Shine, R.; Olsson, M. M.; Moore, I. T.; Lemaster, M. P.; Mason, R. T. 1999. Why do male snakes have longer tails than females? Procedures of the Royal Society of London, 266: 2147-2151.

STATSOFT. 1998. STATISTICA for Windows, Version $5.1 \mathrm{~J}$. Tulsa, USA.

Stebbins, R. C. 1954. Amphibians and reptiles of Western North America. McGraw-Hill, New York, USA, 536pp.

Vincent, S. E.; Herrel, A.; Irschick, D. J. 2004. Sexual dimorphism in head shape and diet in the cottonmouth snake (Agkistrodon piscivorus). Journal of Zoology, 264: 53-59. 